REVIEW ARTICLE

\title{
COVID-19 in Pregnancy: A Clinical Review
}

Rupal Samal ${ }^{1}$, Syed Habeebullah ${ }^{2}$

\begin{abstract}
Novel coronavirus, termed as COVID-19, infection has become a sudden global pandemic, and it is transmitted from human to human. With no evidence-based vaccine or medicine available to treat this viral infection, its incidence and sequelae in antenatal women are debatable, especially in terms of fetomaternal transmission. Pregnant women present with varied flu-like symptoms such as sore throat, throat pain, cough, and fever. They also present with features of dyspnea or shortness of breath. These have been categorized under severe acute respiratory illness per World Health Organization guidelines. Critically ill pregnant women who are COVID-19 positive are monitored and managed with viral pneumonia protocol. The study reiterates judicious use of hydroxychloroquine and antibiotics with proper conservative management. Fetomaternal transmission of COVID-19 is still a controversy, and babies born to COVID-19-positive mothers do not have any potential morbidities. Extensive and stringent care must be provided during antenatal, intrapartum, and postpartum period to ensure transmission-free clinical scenario. The study reemphasizes the fact that it along with maternal and postnatal care, awareness, knowledge, and attitude is essential, and practice on COVID-19 management in pregnancy should be reiterated among all mothers as well as healthcare workers. Proper teaching on hygienic practices, breastfeeding, and postnatal care of the neonates is essential, and mothers should be counseled about the precautionary measures to be followed at the time of discharge.
\end{abstract}

Keywords: Breastfeeding, COVID-19, Newborns, Postnatal care, Pregnancy.

SBV Journal of Basic, Clinical and Applied Health Science (2020): 10.5005/jp-journals-10082-02251

\section{INTRODUCTION}

Novel coronavirus, termed COVID-19, infection has become a sudden global pandemic, where humans are encountering the former as their enemy. The healthcare sector has turned a war zone, where healthcare professionals serve as soldiers. ${ }^{1}$

The COVID-19 infection was first reported in Wuhan domicile, China, in the later part 2019 (reportedly from December 2019). It turned out to be a pandemic and strongly associated with sequelae of adult respiratory distress syndrome (ARDS). ${ }^{1,2}$ The potential lifethreatening virus is known to infect all age-groups, from newborn to old age, with worse outcomes among elderly individuals. ${ }^{2}$

Several other strains of coronavirus that have been already identified and proved to be pathognomonic to humans and manifesting as common cold caused by influenza virus are categorized into middle eastern respiratory syndrome and severe acute respiratory syndrome. ${ }^{2,3}$ Among all the reported strains in Coronaviridae family, the COVID-19 strain has a higher rate of transmission from direct person to person contact by fomites, respiratory tract secretions, and droplets (aerosols). A scientific study revealed that even stool samples show the presence of viral elements collected after a gap period of 29 days after the onset of first symptoms, thus proving to be with longer surveillance compared to other respiratory tract samples. The present observation again reemphasizes the necessity for proper hygiene and safe sanitation. ${ }^{4,5}$

The World Health Organization (WHO) has officially declared COVID -19 to be a pandemic event on mid-March 2020 alarming the world nations. Then onward, it has been recognized as a national emergency worldwide, including VETO-powered countries across the globe, and has initiated measures to contain the spread of this infection. The major challenge of this pandemic is the atypical presentation and varied incidence among different stages of spread in various countries. ${ }^{6}$

\begin{abstract}
1,2Department of Obstetrics and Gynaecology, Mahatma Gandhi Medical College and Research Institute, Sri Balaji Vidyapeeth, Puducherry, India

Corresponding Author: Rupal Samal, Department of Obstetrics and Gynaecology, Mahatma Gandhi Medical College and Research Institute, Sri Balaji Vidyapeeth, Puducherry, India, Phone: +91 9894509492, e-mail: rupalsamal@mgmcri.ac.in

How to cite this article: Samal R, Habeebullah S. COVID-19 in Pregnancy: A Clinical Review. J Basic Clin Appl Health Sci 2020;3(2): 47-52.

Source of support: Nil

Conflict of interest: None
\end{abstract}

COVID-19 in pregnancy is a working concept owing to less experience gained to arrive at conclusions about the incidence of COVID -19 morbidities in pregnant women and newborns, especially in terms of fetomaternal transmissions. ${ }^{6}$

\section{TRANSMISSION}

COVID-19 infection is directly transmitted through humans. The virus can be demonstrated from isolates obtained from respiratory secretions, human feces, etc. Researchers had shown the possibility of vertical transmission (transmission from mother to baby), although the incidence of COVID-19 in pregnancies and its fetomaternal transmission is yet to be documented scientifically. In the present scenario, there is no single reported case of COVID-19 being tested positive from vaginal secretions as well as breast-milk. ${ }^{7,8}$

\section{Effect of COVID-19 on Pregnancy}

Unlike general population, antenatal women do not contact the infection. Generally, during pregnancy, the immune system of the body is altered and also the way it responds to viral infections.

() The Author(s). 2020 Open Access This article is distributed under the terms of the Creative Commons Attribution 4.0 International License (https://creativecommons. org/licenses/by-nc/4.0/), which permits unrestricted use, distribution, and non-commercial reproduction in any medium, provided you give appropriate credit to the original author(s) and the source, provide a link to the Creative Commons license, and indicate if changes were made. The Creative Commons Public Domain Dedication waiver (http://creativecommons.org/publicdomain/zero/1.0/) applies to the data made available in this article, unless otherwise stated. 
Sometimes, they can present as more severe symptoms similar to that of COVID-19. ${ }^{9}$

The mean incubation period (starting from exposure to the virus to the presentation of first clinical features) is noted to be 5 to 7 days. Majority of infected patients will show clinical symptoms farthest by 11 days from the first day of exposure. Travel history especially to abroad countries or direct contact with people who had history of abroad travel in the near past should be monitored. The majority of people both antenatal and general population with COVID-19 infection present with respiratory tract signs and symptoms. ${ }^{10,11}$

Antenatal women present with wide range of symptoms. They present with mild to moderate flu-like illness such as fever, cough, sore throat, and throat pain. Some may show features of dyspnea or shortness of breath. According to WHO guidelines, these women are categorized as severe acute respiratory illness (SARI). Antenatal women, especially those with known history of other medical diseases (diabetes, asthma, etc.), may manifest with symptoms of pneumonia and significant hypoxia. ${ }^{11}$ Vulnerable patients with immunocompromised status and elderly individuals as well as pregnant women may manifests with uncommon symptoms such as easy fatigability, generalized myalgia, malaise, chills, and rigor. Gastrointestinal symptoms such as nausea, vomiting, and diarrhea may or may not be present. Pregnant women with a prior history of congenital or acquired heart disease are at increased risk.

In other types of coronavirus infections such as SARS and MERS, the mother is at a greater risk in the third trimester of pregnancy. There are few case reports of association of preterm birth in women with COVID-19, but it is not clear whether that preterm delivery was always iatrogenic or was spontaneous. ${ }^{12,13}$

The coronavirus pandemic has increased the risk of anxiety and depression in antenatal and postnatal women and also has increased the domestic violence on women. Hence, it is important that mental health of antenatal should be empowered and that women should be asked about her mental health at each and every antenatal checkups. ${ }^{12,13}$

\section{Effect of COVID-19 on the Fetus}

Preliminary research have supported to the fact that the COVID-19 infection does not get transmitted from the mother to fetus through transplacental route or via secretions from the female genital tract. A systematic review on 386 women with COVID-19 showed that there were 257 cesarean sections and 42 vaginal delivery. This relatively higher rate of cesarean sections would reflect the existence of indications for a cesarean section as well as role of fear of vertical transmission. ${ }^{13}$

In a study of mothers who were COVID-19 positive, it was observed among 302 neonates, only 219 neonatal nasopharyngeal specimens were collected and of that 11 samples were positive for SARS-CoV-2. In another study, amniotic fluid samples reported positive for SARS-CoV-2. Two other interesting studies have reported high immunoglobulin $\mathrm{M}$ (IgM) antibody levels in those neonates who were found to be negative for SARS-CoV2 infection. ${ }^{13}$ It was thought to be due to decreased chance of intraplacental transfer of IgM. Furthermore, studies are needed to prove the transmission routes to fetus and the association of maternal COVID -19 infection with child health in the long-term. ${ }^{13}$

There are few studies reporting about the deliveries in COVID -19-positive women. Presently, there are no significant evidence to show the effects on fetus due to infection in terms of major fetal anomalies or other fetal growth parameters, amniotic fluid indices, and Doppler flow indices. There is no validation for endorsing amniocentesis to detect fetal infection in the present situation. At present, there is no confirmed data to suggest increased risk of miscarriage in women with COVID-19 infection. Women with COVID-19 infection are at present not an absolute indication for carrying out medical termination of pregnancy. ${ }^{12,13}$

\section{Criteria for Testing for COVID-19 in Pregnancy ${ }^{13,14}$}

Currently, per ICMR guidelines, each and every pregnant women has to be tested in the any of following circumstances.

- A pregnant woman who presents to OPD with acute respiratory illness and fulfills the following conditions:

- International travel history in the recent past 14 days (dated 06/03/2020 and then on). These individuals with or without symptoms in addition to the testing should also be home quarantined along with their domestic contacts for a minimum of 14 days;

- Is a direct or very close contact of a COVID-positive patient;

- The antenatal women herself is a frontline healthcare worker; or

- Admitted to hospital with signs and symptoms of severe acute respiratory distress.

- A pregnant woman who at present is asymptomatic undergoes testing when they come in contact with direct COVID-positive individuals or high risk contact of a patient who has tested positive. Testing should be done between 5 days and 14 days of contact with these individuals.

- Pregnant women residing in mass population or in containment zones or in large migration gatherings, especially from hotspot districts presenting in labor or likely to deliver 5 days from expected date of delivery, should be tested even if they are asymptomatic.

According to the guidelines published by the Health Ministry and Government of India, direct and high-risk contact is defined as those staying together in the same domicile, traveling together by any means of same transport, working collectively in a same environment with close propinquity, or frontline healthcare workers providing uninterrupted care to the COVID-19-positive patients.

\section{Test Methods and Facilities}

The center of disease control recommends guidelines for nasopharyngeal swabs collection to perform test for COVID-19. In patients with history of productive cough, only sputum should be collected. Sputum induction should not be encouraged. COVID-19 being a RNA virus is identified by reverse transcription polymerase chain reaction (RT-PCR). The RT-PCR is conducted in an NABLaccredited laboratory that is certified by the Government of India and particular State government. At present, the Government of India has allowed RT-PCR to be performed at private laboratories from March 22, 2020. ${ }^{14}$ At present, ICMR recommends RT-PCR as a standard mode of testing. However, 10 to $30 \%$ false-negative tests have been reported to occur even if two serial swabs are tested by the RT-PCR technique. It is due to the presence of known limitations. In future, testing can be conducted by nucleic acid amplification test (NAAT) or by serological testing procedures. NAAT is considered gold standard method of testing. But the major limitation is that the procedure is costly and involves increased risk of viral multiplication. 


\section{Relevant Laboratory Investigations}

Other laboratory investigations that could be carried with COVID19 infection include complete blood counts showing evidence of leukopenia, lymphocytopenia, and mild thrombocytopenia. There will be mild elevation of liver enzymes in liver function tests. Acute markers of infection are also elevated. COVID-19 infections are associated with common cold and common respiratory infections.
Radiological investigations such as computed tomography scan and other radiological imaging modalities usually demonstrate consolidation at periphery of lungs which is also found in pneumonia with atypical presentation. Pregnant women who require an X-ray or a computed tomography scan should be provisioned with an abdominal shield to protect radiation exposure to the fetus. Before doing this, a proper informed written consent per standard guidelines should be obtained.

\section{Management of COVid-19 in Pregnancy (Derived From Lancet) ${ }^{6}$}

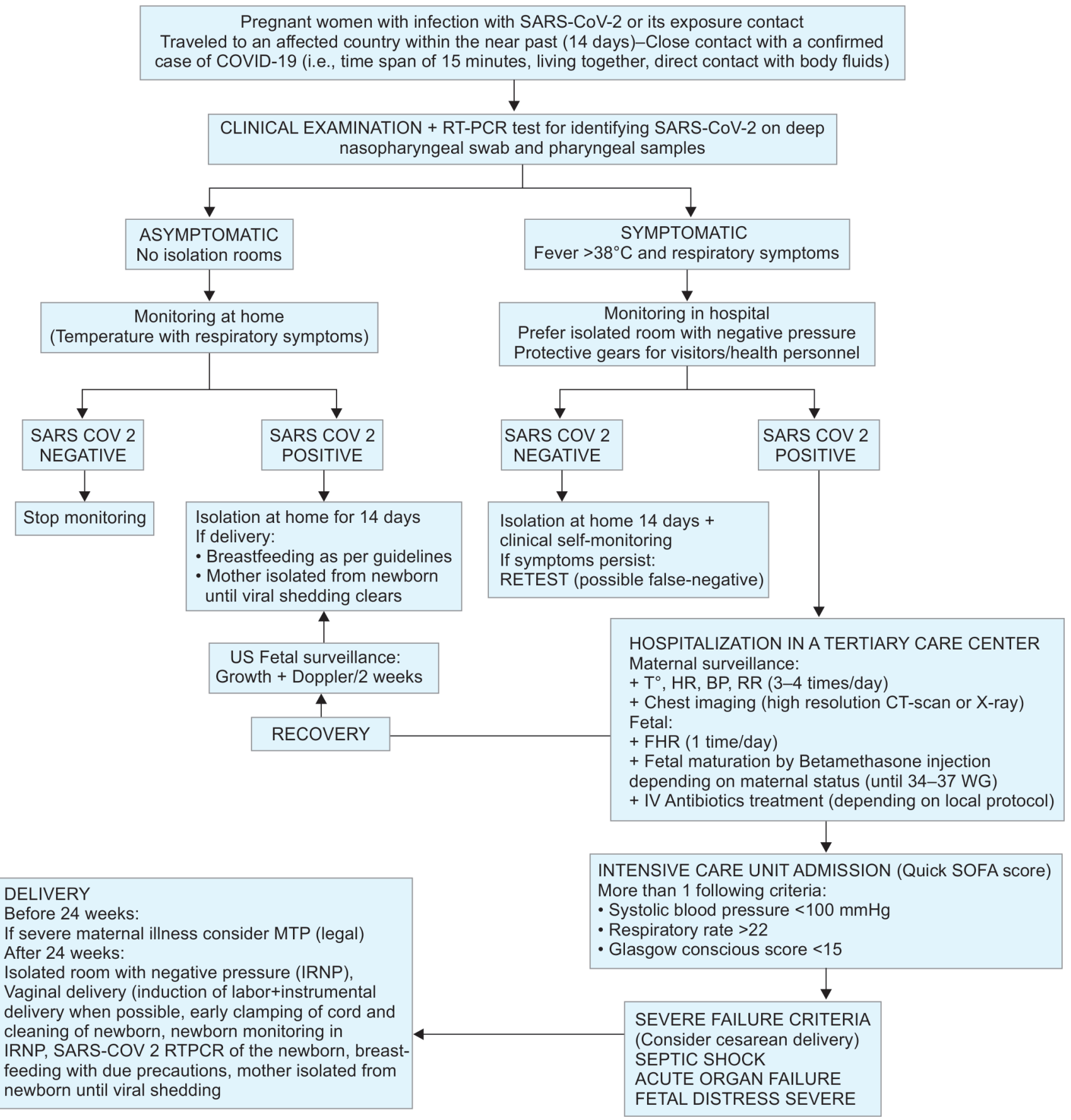




\section{Antenatal Care ${ }^{14}$}

Antenatal women are counseled by maternal care provider to attend routine antenatal checkups with a minimum of four visits at $12,20,28$, and 36 weeks of gestation, unless they are quarantined. In antenatal women having suspected COVID-19 symptoms, appointments for antenatal visits can be deferred for a period of 7 days after the onset of symptoms. Fetal movements and kick counts are to be monitored and maintained. Pregnant women who need to visit the healthcare center must arrange own means of transport and seek the help of 108 ambulance services. Antenatal appointments must be delayed for a period of 14 days who are selfquarantined due to close contacts having symptoms of COVID-19. Pregnant patient whose routine antenatal appointment is delayed for a period of 3 weeks should be contacted by the ANMs or ASHAs pertaining to the particular area. A woman who has formerly tested for COVID-19 negative by laboratory tests, can also manifest with symptoms. Growth scan is advised after a period of 14 days of resolution of COVID-19 infection. ${ }^{13,14}$

\section{Intrapartum Care}

During labor, mother should be quarantined in a private area. A thorough maternal and fetal assessment should be done. A multidisciplinary team including infectious disease specialist or medicine specialist should assess the severity of symptoms. Pregnant women should be delivered in a tertiary-care hospital. Maternal assessment includes respiratory rate, oxygen saturation in room air, and temperature. Fetal assessment is by electronic fetal monitoring using cardiotocograph. Labor onset is confirmed by vaginal examination. ${ }^{15}$

\section{Care in Labor}

The oxygen saturation of laboring women should be targeted to be more than $94 \%$ by titrating the oxygen therapy. Woman showing signs and symptoms of sepsis should be investigated and treated per standard guidelines on sepsis in pregnancy. We should also keep in mind active COVID-19 could be a causative agent as of sepsis and investigate accordingly. Continuous cardiotocograph throughout the labor is recommended. Till date, there is no existing proof that favors vaginal or cesarean delivery. Mode of delivery has no influence on presence or absence of COVID-19 infection but is influenced when the woman's worsening respiratory condition demands immediate delivery. At present, there is no existing evidence whether regional analgesia or anesthesia is contraindicated. In laboring women with confirmed or suspected COVID-19, epidural analgesia is recommended which also can aid in faster delivery if emergency delivery is required. Individualized assessment of the mode of delivery is carried out weighing the risks vs the benefits when there is worsening of woman's symptoms. If emergency cesarean section or operative procedures are performed, then appropriate postpartum examination needs to be done. In symptomatic women who are hypoxic, the labur process, especially second stage, should be cut short with instrumental delivery. ${ }^{16}$

\section{Critical Care Management of Patients WITH COVID-19 17}

Pregnant women with the following criteria:

- Respiratory rate $>30$ per minute

- Oxygen saturation $<93 \%$ at rest
- Partial pressure of oxygen $\left(\mathrm{PaO}_{2}\right)$ arterial/oxygen concentration $\left(\mathrm{FiO}_{2}\right)<300 \mathrm{~mm} \mathrm{Hg}$

- Lung imaging showing $>50 \%$ lesions progression within 24-48 hours

- Quick sequential organ failure assessment score (qSOFA) score has been an adjunct tool in decision-making for critical care management.

A quick bedside assessment tool is also usable for sepsis (typically for bacterial infections) screening in triage called the "quick SOFA (qSOFA) score" (Table 1). One point is included for each of 3 criteria. $^{18}$

Vitals are observed hourly, and the trends are monitored. Oxygen saturation is titrated to be maintained more than $94 \%$. Respiratory rate is monitored hourly. Young age-group women with healthy status can also have worsening of respiratory symptoms but will be able to sustain a normal oxygen saturation before they abruptly decompensate. Hence, respiratory rate will increase with maintenance of normal saturation which also indicates worsening of pulmonary function and should be treated by starting or increasing the nasal oxygen flow rate. Radiological investigations can be accomplished based on priority among nonpregnant women, which includes chest $\mathrm{X}$-ray and computed tomography scanning of thorax that should be performed instantly for fetal concerns. Proper abdominal guarding using shield should be used to safeguard the fetus per standard guidelines. Ancillary investigations such as ECG, CTPA as appropriate, and echocardiogram can also be done simultaneously. Care should be taken not to presume that all febrile illness are because of COVID-19 infection. Chance of bacterial infection should be suspected if the white blood cell count is elevated (lymphocyte count will be in normal range or decreased) and then to start appropriate antibiotic treatment. Stringent precaution is to be observed in case of intravenous (IV) fluid management. An attempt of fluid boluses of $>250-500 \mathrm{~mL}$ volume is given and then assessed for evidence of fluid overload before starting any resuscitation with IV fluids. In case of emergency, delivery process has to be initiated. In view of indications pertaining to fetal causes, delivery should be conducted via normal as long the mother remains stable. ${ }^{17}$

Critical antenatal women with COVID-19 infection should be managed per the guidelines of viral pneumonia. The basic principle for managing ARDS includes the following:

- Intravenous fluid management

- Start of empirical antibiotics with a concern to manage possibility of pneumonia due to bacterial etiology

- Early ventilator support for respiratory assistance may be provided

- Protective lung ventilatory measures, and

- Interval repositioning..$^{17}$

Table 1: Quick SOFA score (qSOFA)

\begin{tabular}{|c|c|c|c|c|}
\hline \multicolumn{4}{|c|}{ qSOFA SCORE } & \\
\hline Number & Criteria & & Point & \\
\hline 1 & $\begin{array}{l}\text { Respiratory } \\
\text { rate }\end{array}$ & $\begin{array}{l}\geq 22 \text { breaths/ } \\
\text { minute }\end{array}$ & 1 & $\begin{array}{l}\text { Score } \geq 2 \text { is } \\
\text { suggestive of sepsis } \\
\text { and needs intensive } \\
\text { care number }\end{array}$ \\
\hline 2 & Mental status & Altered & 1 & \\
\hline 3 & $\begin{array}{l}\text { Systolic blood } \\
\text { pressure }\end{array}$ & $\leq 100 \mathrm{~mm} \mathrm{Hg}$ & 1 & \\
\hline
\end{tabular}




\section{Medical Management of COVID-19 Infection in Pregnancy 9,10}

Initial supportive management includes adequate bed rest, proper supplementation of nasal oxygen and as well as nutritional supplements.

Two modalities of treatment has been attempted for COVID-19 infection. Combination of hydroxychloroquine and azithromycin stands as the treatment modality that is cheap and easily available in the country. Antiviral drugs stand as the second modality that is not standardized in India. ${ }^{17}$

Hydroxychloroquine should be given at a dose of $600 \mathrm{mg}$ or 200 mg three times a day after food) and azithromycin (500 mg once a day) for a period of 10 days. ${ }^{9}$ Similar combination of medications was attempted for treatment of 20 patients with symptoms of upper and lower respiratory tract infections with an exemption of pregnancy patients. ${ }^{19}$ However, the combination of these drugs have proved to cause no significant adverse effects on mother and fetus when used antenatally or during lactation. Hydroxychloroquine can also be supplemented in an alternate dosage such as $400 \mathrm{mg}$ twice daily on first day and then $400 \mathrm{mg}$ once daily for the next 4 consecutive days. Studies recommend adding azithromycin only when there is additional bacterial infection. ${ }^{19}$

\section{Antiviral Treatment}

The first antiviral drug used in combination to manage COVID19 infection globally was Lopinavir-ritonavir. This therapy was considered as the first-line management for patients suffering from long-term illness, immunosuppression, or uncontrolled diabetes. In India, a section of health official panel have advised Oseltamivir 75 mg twice daily for 5 days in combination with hydroxychloroquine. This suggestion of drug is given based on evidenced obtained from experience of the H1N1 (swine flu) outbreak. However, the available data on this regime are very sparse, although it proved to be a simple and cost-effective drug. ${ }^{2,19}$

\section{Vaccine}

In the present scenario, various public and private sectors are doing scientific research to develop and formulate a vaccine. It is anticipated that a potential evidence-based vaccine would be marketed after a period of 10 to 14 months..$^{3,19}$

\section{Other Drugs}

\section{Nonsteroid Anti-inflammatory Drugs (NSAIDs)}

The most commonly used drug used for symptoms of fever and myalgia. Tablet paracetamol is considered as the drug of choice.

\section{Antenatal Steroids (Fetal Maturity)}

Antenatal corticosteroids are used for fetal lung maturity, where gestational age is between 24 weeks and 34 weeks of gestation.

\section{Antihypertensive}

There is debate prevailing on the use of angiotensin-converting enzyme inhibitors and angiotensin receptor blockers in older patients with hypertension. These drugs are avoided in pregnancy due to teratogenic effects. ${ }^{4}$

\section{Antibiotics}

Antibiotics are prescribed when there is doubt for presence of secondary bacterial infection.

\section{Oxygen}

In the event of dyspnea, oxygen supplementation with nasal prongs or mask should be done. High flow oxygen either by nasal prongs or by nasal masks should be started instantly at a rate of 4 to $6 \mathrm{~L}$ per minute. Noninvasive ventilation can also be used based upon the clinical situation. Regular close reevaluation of the patient's status should be done, and shifting to intensive care units should be considered based on progression. ${ }^{5,19}$

\section{Postnatal Management}

In the present scenario, there is no clear evidence on cards that the newborns of COVID-19-positive mothers are at a higher risk of worsening complications. There is a growing concern that the infant may get infected after delivery when they come in contact with respiratory secretions of COVID-19-positive mother. The healthcare team members should discuss the benefits and risks of temporary separation of mother and baby. An isolation room that is private should be set up for the newborns. The decision to temporarily separate the mother from her baby is decided on a clinical basis with consultation from clinicians, infection control authorities as well as public health officials. ${ }^{7,8}$ Collocation ("rooming in") of the newborn is decided per mother's decision. Other simple infrastructure arrangements such as physical barriers using screen or curtains and keeping the newborn at least 6 feet distance away from the ill mother should be made. The mother should use sterile facemask each and every time when in contact with the newborn. These practices should be stringently continued till the mother discharged from the hospital. ${ }^{10,18}$

\section{LACTATION}

During the transient separation period of mother from fetus, breastfeeding can be carried out by encouraging the mother to pump breast-milk by pumping/manual squeezing to establish the flow of breast-milk production and to maintain milk supply. A specially devised breast pump can be used. ${ }^{11,12}$ However, proper hygiene and precaution must be encouraged before taking the breastmilk. At the end of each pumping event, all the parts involved in direct contact with breast-milk must be stringently washed, and disinfection procedure must be carried out per the manufacturer's instructions. ${ }^{13}$ The breast-milk pumped and collected must fed to the neonates with the aid of an experienced caregiver in healthy status. However, due to the known psychological discomfort that occurs postnatally and if a mother intends to have room-in and wants to carry on direct breastfeeding, proper facemask, gloves, and aseptic precautions including hand hygiene must be ensured. ${ }^{14,15}$

\section{Hospital Discharge}

Discharge process of the postnatal mother must be initiated only when the standard tests proved to be negative, and the health condition of both the mother and the newborn is stable. The discharge summary issued to the patient must definitely describe the advice about precautionary measures and guidelines to be followed for COVID-19 infection, with a special reiteration on social distancing practices and enriching awareness on acute respiratory illness. ${ }^{16}$

Discharge of postpartum women should be done when they test negative and maternal and fetal/neonatal condition 
is stable. ${ }^{16,17}$ The discharge card from the maternity unit should contain precautions about COVID-19 infection in addition to the usual postdelivery instructions. It should also emphasize social distancing and need for evaluation if symptoms of SARI arise after delivery. ${ }^{18-20}$

\section{Conclusion}

The study reemphasizes the fact that it is essential along with maternal and postnatal care, awareness, knowledge, attitude, and practice on COVID-19 management in pregnancy should be reiterated among all mothers and healthcare workers. Proper teaching on hygienic practices, breastfeeding and postnatal care of the neonates and mothers is essential, and mothers should be counseled about the precautionary measures to be followed at the time of discharge.

\section{References}

1. Team NCPERE. Vital surveillances: the epidemiological characteristics of an outbreak of 2019 novel coronavirus diseases (COVID-19) - China. Team NCPERE. China CDC Weekly 2020;8:113-122.

2. Wu Y, Guo C, Tang L, Hong Z, Zhou J, Dong X, et al. Prolonged presence of SARS-CoV-2 viral RNA in faecal samples. Lancet Gastroenterol Hepatol 2020;5(5):434-435. DOI: 10.1016/S2468-1253(20)30083-2.

3. World Health Organization, General-s-opening-remarks-at-themedia-briefing-on covid-19. 11-March-2020.

4. FOGSI NNF India IAP. Perinatal Neonatal Management of COVID-19 Infection. New Delhi: 2020.

5. COVID19 (nCorona) Virus Outbreak Control and Prevention State Cell. COVID-19 Advisory for Pregnancy and Labour Management. Health and Family Welfare Department, Government of Kerala. [Online] March 24, 2020. [Cited: March 28, 2020.] http://dhs.kerala.gov.in/ wp-content/uploads/2020/03/labour_24032020.pdf.

6. The Lancet. COVID-19: protecting health-care workers. Lancet 2020;395(10228):922. DOI: 10.1016/S0140-6736(20)30644-9.

7. Royal College of Obstetricians \& Gynaecologists. Coronavirus (COVID19) infection in pregnancy guidelines. 2020;26(03):117-126. DOI: 10.1093/humupd/dmz040

8. World Health Organization, Global Surveillance for Human Infection with novel Coronavirus. 2019.
9. Indian Council for Medical Research. COVID-19 testing. [Online] [Cited: March 28, 2020.] https://www.mohfw.gov.in/pdf/Advisoryontheuse ofHydroxychloroquinasprophylaxisforSARSCoV2infection.pdf.

10. EMR Division, Ministry of Health and Family Welfare, Government of India. Guidelines on Clinical Management of COVID-19 infection. [Online] https://www.mohfw.gov.in/pdf/ Guidelines on Clinical Management of COVID 19 12020.pdf.

11. Tang B, Xia F, Tang S, Bragazzi NL, Li Q, Sun X, et al. The effectiveness of quarantine and isolation determine the trend of the COVID-19 epidemics in the final phase of the current outbreak in China. Int J Infect Dis 2020;95:288-293. DOI: 10.1016/j.ijid.2020.03.018.

12. Chen H, Guo J, Wang C, Luo F, Yu X, Zhang W, et al. Clinical characteristics and intrauterine vertical transmission potential of COVID-19 infection in nine pregnant women: a retrospective review of medical records. Lancet 2020;395(10226):809-920. DOI: 10.1016/ S0140-6736(20)30360-3.

13. Mirbeyk M, Rezaei N, The impact of COVID-19 on pregnancy and neonatal health: a systematic review, 01 May 2020, PREPRINT (Version 1) available at Research Square [https://doi.org/10.21203/rs.3.rs25861/v1+].

14. Favre G, Pomar D, Musso D, Baud K. nCoV-2019 epidemic: what about pregnancies? Lancet 2020;395(10224):e40. DOI: 10.1016/S01406736(20)30311-1.

15. Gautret $\mathrm{P}$, Lagier JC, Parola $\mathrm{P}$, Hoang VT, Meddeb L, et al Hydroxychloroquine and azithromycin as a treatment of COVID-19: results of an open label non-randomized clinical trial. Int J Antimicrob Agents 2020;20:105-115. DOI: 10.1016/j.ijantimicag.2020.105949.

16. Cao B, Wang Y, Wen D, Liu W, Wang J, Fan G, et al. A trial of lopinavirritonavir in adults hospitalized with severe COVID-19. N Engl J Med 2020;18(19):39-45. DOI: 10.1056/NEJMoa2001282.

17. Ministry of Health and Family Welfare, Government of India. Guidelines for notifying COVID-19 affected persons by private institutions. [Online] [Cited: March 28, 2020.] https://www.mohfw. gov.in/pdf/GuidelinesfornotifyingCOVID-19 affected persons by Private Institutions.pdf.

18. Huang C, Wang X, Li L, Ren J, Zhao Y, Hu L. Clinical features of patients infected with 2019 novel coronavirus in Wuhan, China. The Lancet 2020;395(10223):497-506. DOI: 10.1016/S0140-6736(20)30183-5.

19. Yang $\mathrm{H}$, Wang $C$, Poon LC. Novel coronavirus infection and pregnancy. Ultrasound Obstet Gynecol 2020;55(4):435-437. DOI: 10.1002/ uog.22006.

20. The First Affiliated Hospital, Zhejiang University School of Medicine. Handbook of COVID-19 Prevention and Treatment. Wuhan: Jack Ma Foundation, 2020. https://covid-19.alibabacloud.com/. 